\title{
FILM SHOWING THE TECHNIC OF DENTAL PROPHYLAXIS FOR THE DENTAL HYGIENIST.
}

\author{
By Alfred C. Fones, D. D.S., Bridgeport, Conn.
}

(Read before the National Dental Association at Its Twenty-First Annual Session, New York City, N. Y., October 23-26, 1917.)

$\mathrm{T}$ O AID in the great cause of mouth hygiene the moving picture has been resorted to for illustrating some of the phases of the work, and altho our efforts are rather crude in comparison with the teaching films that will be created in the future yet they show that the pictures are helpful in conveying to the minds of the audience the work itself and the technic employed.

To teach the operative side of dental prophylaxis to students by lectures is exceedingly difficult, and altho the divisions of instrumentation and polishing can be memorized, the most important of all is to see the actual motion of the instruments. This, in the past, we have been obliged to illustrate from the platform and by personal demonstration to each student.

In teaching this work to students the stereopticon should first be used to show the position of the hand for division 1 in instrumentation, noting the fulcrum point, the grasp of the instrument, the teeth involved, the surface to be covered and the motion employed. After the picture has been carefully analyzed, the motion picture will show the play of the instrument on the surfaces of the teeth. Then the student may turn to the manikin and assume the correct position of hand and instrument and attempt to simulate the motion. The instructor should then pass from chair to chair to see that each student has thoroly comprehended what he has seen.

Each division is thus slowly presented, the student going over and over it until he is able to do it well.

In presenting this teaching film today for your criticism I would have you understand that the technic employed is for an ordinary prophylactic operation. Surgical instrumentation at the present time is beyond the province of the dental hygienist and is not shown on this film. But when heavy deposits are present, as in a first cleaning, the sickle shaped instrument may be used by the hygienist to remove the large accumulation of calculus.

There are four distinct motions used in instrumentation and polishing; digital wrist, forearm or rotary, and rigid arm. The student must first learn these four motions. As much of the work is done with the rotary stroke, a prolonged practice should be made of this one motion.

Opinions vary so much among operators and teachers concerning instruments, and there is so little yet standardized in dental operations, that the instruments selected to perform this work may not be generally accepted. We have tried many scalers and have been able to secure the best results with the instruments shown here. These small instruments used on the lingual and buccal surfaces are the Darby-Perry excava- 
tors, Nos. 17 and 18. Their small size greatly magnifies the sense of touch, and small deposits are thus quickly detected. In using the blade at the angle shown there is but little danger of wounding the gums, and the back of the blade, rapidly patting the gingivae, squeezes

Figure 1.

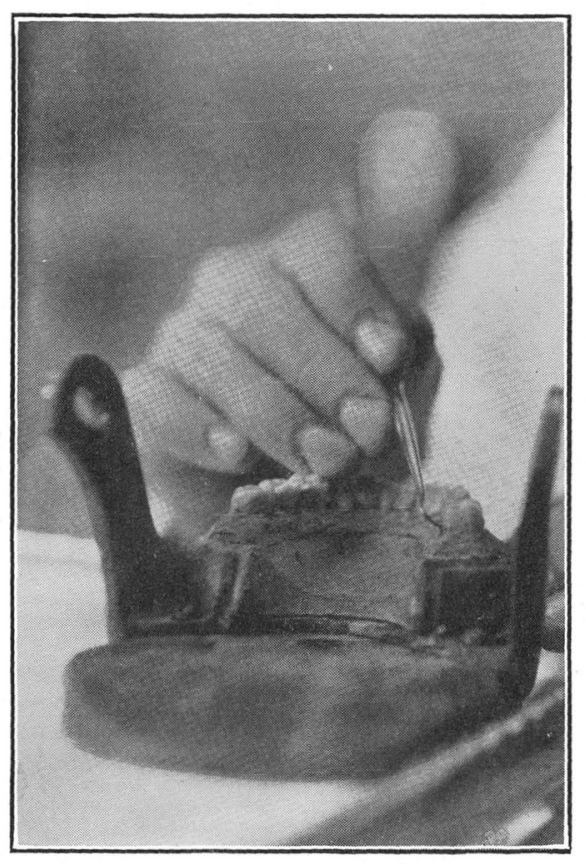

A typical illustration from the film during instrumentation showing instrument used, with proper hold, adaptation and fulcrum point of hand.

the blood out of the congested surfaces and acts as a massage to the borders of the gum tissue.

The base of each tooth has four lines. The buccal and lingual lines having been covered, the distal and mesial surfaces are scaled by using instruments Nos. 13 and 14 of the Smith set. These are file cut, with numerous sharp blades and are very effective in reaching between the teeth and approximal surfaces.

The artist, when asked how he secured such wonderful colors, remarked that he mixed his paint with brains.

These instruments are not forced between the teeth when the gum tissue is normal and fully occupies the interproximal space, but unfortunately, in the majority of the mouths of adults, the gum tissue in these spaces shows a shrinkage and congestion that will permit of at least the partial introduction of these instruments. The approximal surfaces of incisors are scaled by using the bayonet shaped instruments, Nos. 5 and 6 of the Smith set.

While the film is showing a similar operation on the upper jaw, let us consider two special features under instrumentation. First, the sensitiveness that is frequently found around the necks of the teeth, and second, the bleeding of the gingival borders of the gums. In adults, where the lime deposits have been heavy, their removal will frequently cause much sensitiveness for a week or two, sometimes even longer, to heat and cold and to sweets and acids. The deposits have caused a destruction of the borders of the gingiva, the cementum and the soft tissues around the necks of the teeth, exposing the ends of the fibrils in the granular layer between the dentin and the cementum and forming an area that is highly sensitive to the touch of the instrument or polisher. It is frequently wise to inform the patient that he may expect the surfaces to be sensitive to heat and cold for a short time, in order to allay any fears on his part. The deposits acting as a covering for the surfaces, have protected them from external irritation and the patient is apt to wonder why it is that his mouth is so much more sensitive than it was before the deposits were removed. Acids are especially irritating to these surfaces, and the use of bicarbonate of soda, half a teaspoonful to a third of a glass of warm water used as a mouth wash two or three times daily, will greatly aid in tiding over this short period of discom- 
fort. If the soda can be used clear by dipping the finger in water, touching it to the soda and then rubbing it on these surfaces, it will all the more quickly neutralize any acid that may be irritating to this sensitive tissue. The thoro rubbing and polishing with the stick and pumice and the extreme cleanliness from the faithful use of the tooth brush will soon bring these troublesome areas under control. A ten per cent solution of nitrate of silver is some times applied by the operator, but if it is used it should be followed by a thoro polishing with the stick and pumice.

The second feature, which is considered briefly, is the bleeding of the gums during instrumentation. When the gums readily bleed there is congestion of the capillaries, and the more blood allowed to escape from the gingivae, the sooner the congestion will be relieved. Instead of trying not to make the gums bleed, just the reverse course should be followed altho of course this does not mean that they should be lacerated or the tissue wounded.

The bleeding process is produced by using the back or smooth surface of the blade of the instrument with pressure, and this is done while removing the lime deposits. If there should be a copious flow of blood from some of the approximal surfaces it should be encouraged with rapid gentle pressure strokes directly on the gingivae. Healthy gums will not bleed during instrumentation, and when bleeding occurs enlarged and congested capillaries are sure to be found. No fear of causing injury to the gum tissue in causing a flow of blood need be felt as long as care is taken that the blade of the instrument does not cut the tissue. Frequently after such a treatment the gums will take on a color two shades lighter before the patient leaves the chair, and after a few days of stimulation with the tooth brush it will be hard to recognize it as the same deep red, congested tissue that it was to begin with.

\section{PoLISHING.}

It is impossible to obtain the same results in prophylaxis with the use of the dental engine in polishing as may be secured with the hand polishers. This belief is based upon personal experience in faithfully trying out both methods and is an accepted fact by all prophylactic workers who have become proficient with the hand polishers.

The object of this polishing process is threefold. First, the removal of stains, placques or films, or all soft accretions on the exposed surfaces of the teeth. Second, a polishing of the enamel surfaces and a stimulating effect that seems to be imparted to the living tissue of the toothe itself by the vigorous massage. Third, the beneficial results obtained on the gingival borders of the gums by the slight bumping of the stick, causing light pressure and release which imparts a massage effect and aids greatly in producing a perfect flow of blood thru the capillaries in the peripheral circulation. If a new case presents itself in which the teeth are badly stained, it is perfectly reasonable, if desired, to use the dental engine for the first treatment to aid in cleaning off these stains from the enamel surfaces, but all subsequent treatments should be made with the hand polishers. An engine revolving at six or eight hundred revolutions a minute, with the rubber cup or buff charged with pumice, cuts too vigorously, and if used at each prophylactic treatment, will in time effect the enamel and tooth structure at the necks of the teeth. With the dental engine all sense of touch is lost, and besides it is not as adaptable on the approximal surfaces or on the surfaces of the molars as the stick held in the hand. The gingival borders of the gums, in many mouths, have been badly wounded or damaged by the revolving cups or buffs in the dental engine, and if one hopes and expects to secure the best results in obtaining ideal health 
conditions of these tissues, one must become proficient with the hand polishers. Those who would advocate the dental engine are those who have failed to make themselves proficient with the hand polishers. There can be no choice if the latter is faithfully tried.

Figure 2.

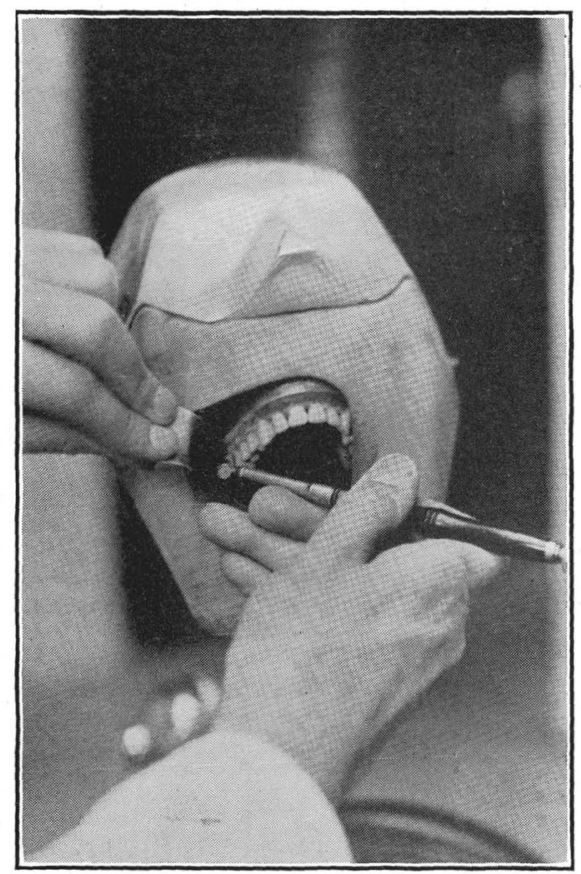

An illustration from the film during polishing, showing adaptation of large stick and cheek distender.

There are a number of different woods that may be used for polishing, as cedar, maple, hard pine, etc., but the closest grained wood and the one best adapted for this purpose is orange wood. There are two sizes of sticks that may be had from the dental depots, known as large and small. The large size is cut about three-quarters of an inch in length and one end is cut wedge-shaped. This stick is used on all the broad surfaces of the teeth except the masticating surfaces.
The small stick is cut about the same length and one end is cut like the point of a lead pencil. The smaller stick is used on the approximal surfaces and around the necks of the teeth where it is impossible to adopt the larger stick.

In order to work with facility two holders for the two sizes of sticks should be employed.

As a slight abrasive and polish to be used with the sticks, the finest grade of pumice moistened with water will prove to be most satisfactory. Altho other polishing mediums are used with good results, it is doubtful if there is anything superior to fine pumice for this special work. A scant spoonful in a small porcelain dish, and wet sufficiently with water to be almost liquid, will make a mixture that can be readily picked up on the point of the wet stick and used in the mouth.

It is impossible to reach with the polishers the contact points and an area on the approximal surfaces of the teeth. As a large percentage of all dental decay takes place on these surfaces they require the most careful attention. A strand of ligating silk, doubled and twisted, is charged with pumice and carefully passed between the contact points and rubbed back and forth on the mesial and distal surfaces of the teeth. Where the space between the teeth permits Cutter's wide floss may be used to advantage.

The masticating surfaces are so uneven that they cannot be polished well with a stick and it becomes necessary to use a brush wheel in the engine for this purpose.

The treatment just described is for an adult mouth, it being seldom necessary to use instruments for children. The same method of polishing is followed in the prophylactic treatment of children. 
Home Care of the Mouth. Brushing.

Ideas are somewhat varied as to the proper way of brushing the teeth and

Figure 3.

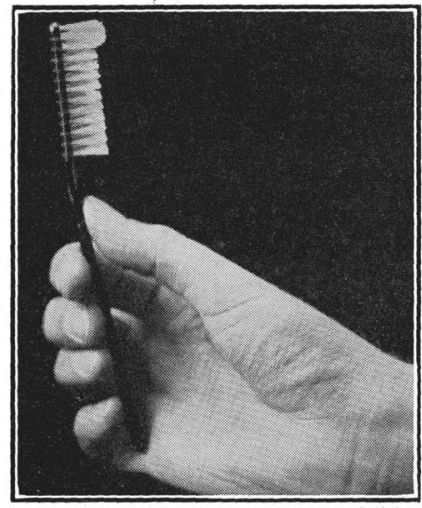

Hold of brush for cleansing outside surfaces of the teeth and gums, left side. The cut of the bristles is especially adaptable for a combined tooth and gum brush.

gums. A Japanese once said in reference to religions that all roads lead to

Figure 4.

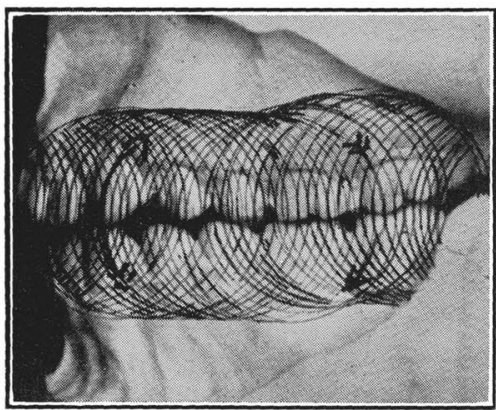

Showing circular motion given brush on the left side and front of the mouth on teeth and gums. A light rapid stroke of the brush is used at all times. Include both upper and lower gums in the largest eircle it is possible to make.

the top of the mountain, yet some seem shorter than others. Several of the methods employed in cleaning the teeth and massaging the gums are helpful and will produce good results, but I have found that the method here advocated has been the most helpful and satisfactory to us

Figure 5.

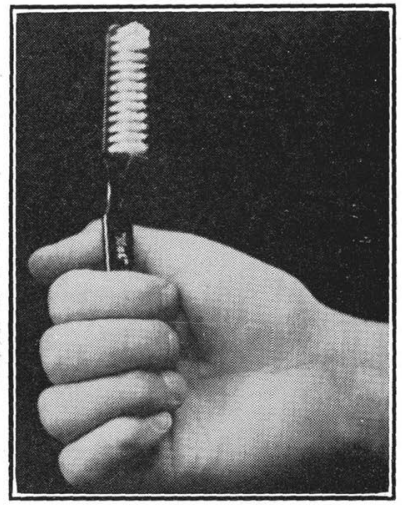

Hold of brush for right side in brushing outside surfaces of the teeth and gums.

and to our patients. I have been using this method of brushing in my practice since the winter of 1905 and am confident that after twelve years of careful

Figure 6 .

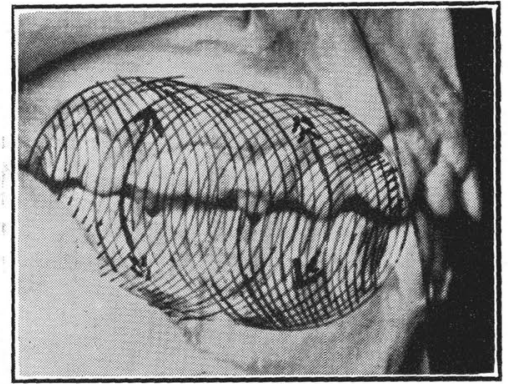

Circles in which brush should travel on right side.

observation that it will live on for many years to come, and prove to be exceedingly helpful in our work for mouth hygiene.

In teaching the method of circular brushing the beginner should be instructed to use a light stroke, little or 
no pressure being applied by the bristles on the gums until the mucous membrane has had an opportunity of thickening and producing an armor for the

Figure 7 .

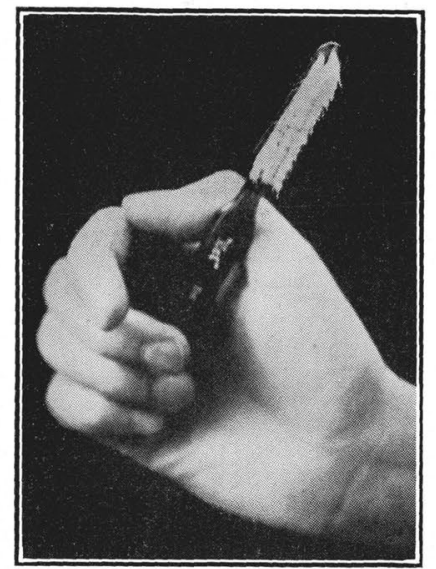

Hold for brushing inside surfaces of lower teeth and gums. It is not necessary to use pressure with the brush at any time.

underlying tissues. This thickening process takes place in a few weeks and is analogous to the results produced on

Figure 8.

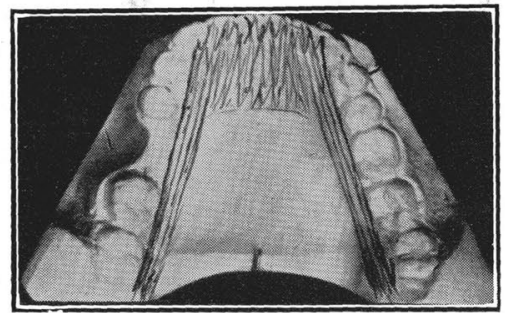

Lines indicating the direction the brush should travel with an in-and-out stroke, using chiefly the tuft of the brush.

the skin of the hands or body when subjected to friction each day. The gums take very kindly to this form of brushing, the stimulus imparted to the underlying tissues being very noticeable, the gingivae becoming tough and firm and the color of the membrane changed from red to a pinkish hue.

\section{FLOSSING.}

There is but one way that is effective

Figure 9.

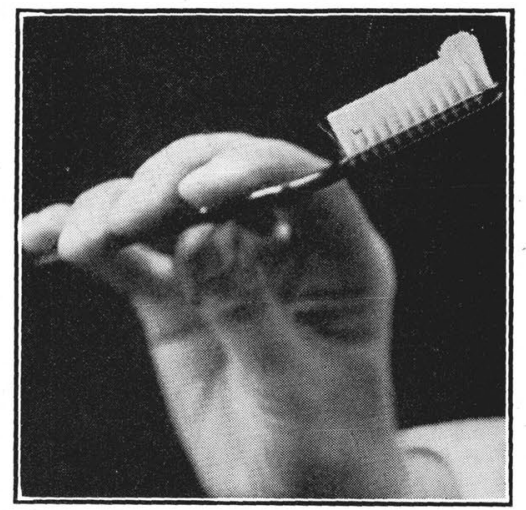

Hold of brush for inside surfaces of upper teeth and roof of mouth.

in removing the food from between the teeth, and that is with a piece of dental floss. The floss should be forced care-

Figure 10.

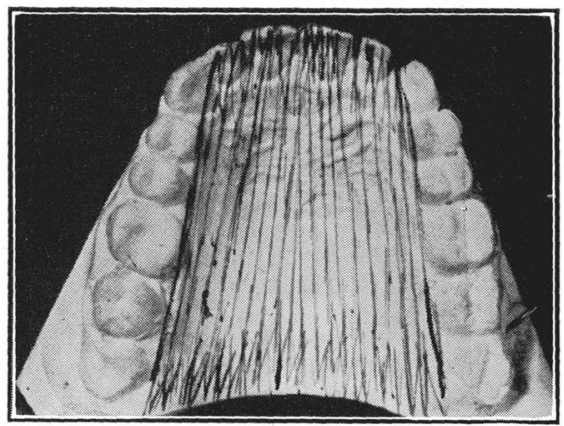

Showing the direction the brush travels with the in-and-out stroke. No illustrations are necessary to show the cleansing of the grinding surfaces of the teeth and distal surfaces of last molars. The hold and motion most convenient may be used.

fully between all the teeth, care being exercised not to injure the gums in so doing. It should be rubbed back and 
forth against the surfaces of each tooth to clean them.

Lime Water Mouth Wash.

Of the many prepared mouth washes

Figure 11.

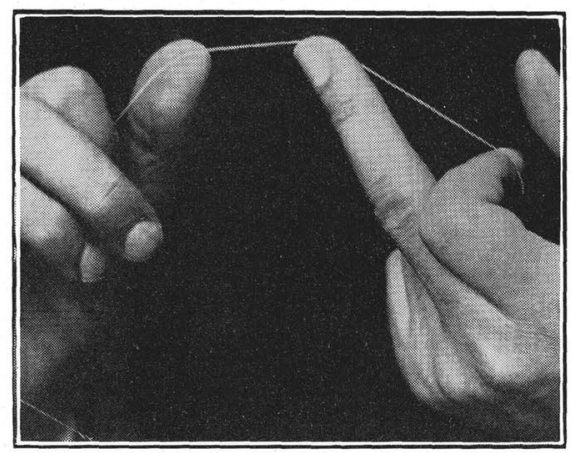

Hold of floss for right upper teeth.

Figure 12 .

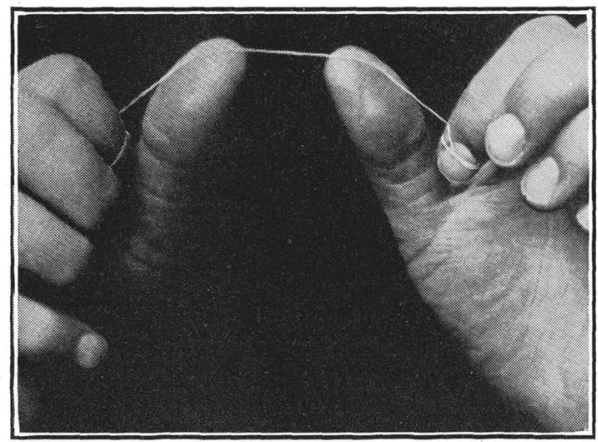

Hold of floss for left upper teeth.

advocated, we believe that a mouth wash made from coarse calcium oxide is one of the most efficient. The lime water thus prepared has the power of dissolving the precipitated mucin from the surfaces of the teeth thus destroying the bacterial placque. Altho we cannot realize our highest hopes in the use of

Figure 13.

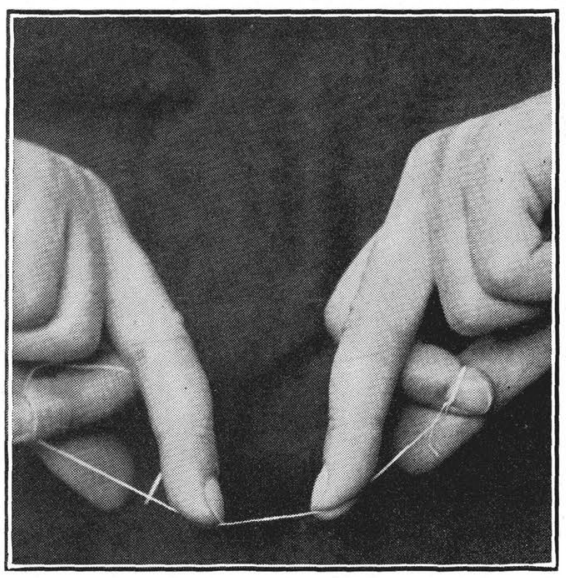

Hold of floss for lower teeth.

lime water as a perfect solvent for the mucilagenous placques, yet it is about as efficient as anything we know of at the present time. One-half cupful of the powdered coarse calcium oxide in a quart of cold water is the correct proportion for use. 